\title{
Exploring the Utility of Remote Messaging and Situated Office Door Displays
}

\author{
Keith Cheverst, Alan Dix, Dan Fitton, Adrian Friday and Marc Rouncefield \\ Department of Computing, \\ Lancaster University, \\ Lancaster, LA1 4YR. \\ e-mail: kc,dixa,df,adrian@comp.lancs.ac.uk and m.rouncefield@lancaster.ac.uk
}

\begin{abstract}
In recent years, the proliferation in use of the GSM short message service (or SMS) has prompted numerous studies into person to person messaging via mobile devices. However, to date, there has been relatively little exploration of systems that enable mobile messaging to (potentially ubiquitous) situated displays rather than the mobile devices of particular individuals. In this paper, we describe the results of an ongoing trial to explore the utility of a system that enables lecturers in a computing department to use their mobile phones to send messages to digital displays situated outside their offices.
\end{abstract}

\section{Introduction}

Perhaps one of the most surprising phenomena to have occurred within the field of mobile computing within recent years has been the uptake of SMS (or Short Message Service) text messaging. For example, according to the Mobile Data Association (MDA), the total number of chargeable person-to-person SMS text messages sent across the four U.K. GSM network operators during the day between midnight on 31st December 2002 and midnight on 1st January 2003, was 102 million, a 59\% increase compared to figures over the same period in 2002 [3].

One use of SMS that has received little investigation to date is to enable a (potentially mobile) user to message a display in a particular place rather than a mobile device owned by a particular individual. O'Hara et al [4] discuss situated displays as being "situated at particular locations within our environment" and yet which, paradoxically, "allow information to be more easily updated dynamically and remotely". Although the origins of our work pre-date this quote, it is exactly this potential for updating situated displays remotely that forms the focus of this paper.

The Hermes system [2] was designed to explore the issues that arise when office owners are provided with a digital 'office door display'. One issue we wished to explore was whether the traditional way of posting messages on post-it notes in 'semiprivate' places could be enhanced with such a digital equivalent. One of the major gains of an electronic system is that it supports remote interaction. This includes a web-based interface, email and, most significantly for this paper, SMS. 
There are numerous examples of office door display systems and a full overview of these and related systems can be found in [2]. However, none of these currently provide (much less evaluate) an explicit facility for mobile messaging. One interesting example of a system that utilises SMS messaging in conjunction with situated display involves a U.K. police force. This police force has developed a system that enables appropriate personnel to use SMS in order to post road warnings to displays situated on the roadside [1]. In common with Hermes, the system is designed to supplement existing approaches. In this case, the existing approach for alerting anonymous drivers of hazardous road conditions is local radio.

\section{A Brief Overview of the HERMES System}

Work on developing the Hermes system started in October 2001 and the first unit was installed outside one of the offices in the computing department in March 2002. Currently ten Hermes displays are deployed around the department outside the offices of lecturers, research assistants, $\mathrm{PhD}$ students and department secretaries. Figure 1 illustrates a Hermes display and one of the department's corridors containing a concentration of Hermes displays.
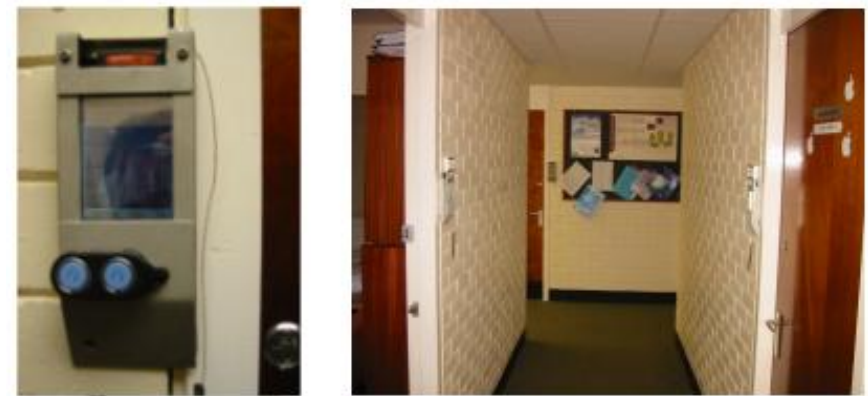

Figure 1: Picture of an early Hermes display (left) and deployed displays (right).

The functionality supported by the system can be considered from two main perspectives, namely: the perspective of the owner of the Hermes display and the perspective of a visitor to the Hermes display.

Owners can create messages to appear on their own Hermes displays. Messages (which can take the form of text or graphics) can be created on the display itself, via the Hermes web page, an e-mail client or by sending an SMS text message. This message is then visible on the door display.

One important aspect of a Calm technology is that it "...engages both the center and the periphery of our attention, and in fact moves back and forth between the two" [5]. The Hermes display has just these properties: for a passer-by it does not grab attention, but for someone visiting the office it is immediately obvious. The situated nature gives us this flexibility of attention 'for free'.

Visitors can also leave messages on an owner's display. However, once entered, messages left by visitors are not visible to passers-by. There are two main reasons for controlling this aspect of visibility. Firstly, because of the limited display space, 
Hermes must give priority to the owner's message. Secondly, we wanted to encourage owners to feel in control of the personal space represented by their individual Hermes displays. The owner can read left messages via a web portal, email or using his or her Hermes display directly. Owners can also request notification via SMS when a new message is left on their Hermes display.

A sketch illustrating the basic system architecture of Hermes is shown in Figure 2. A detailed explanation of the architecture is beyond the scope of this paper, but more details of the approach can be found in [2]. Briefly, at the heart of the system is a single central server application that stores messages and user profile information, hosts the Hermes web portal and supports communication with the SMS Gateway.

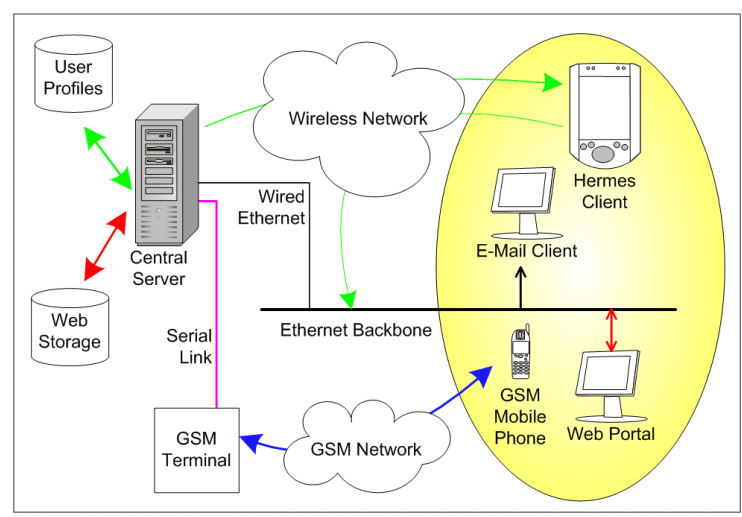

Figure 2: The System Architecture of Hermes [x].

\section{Motivation for Enabling "Texting" to Hermes Displays}

From an early design stage we realized the potential importance of providing the owners of Hermes displays with the ability to send a message to the display situated outside their office using their mobile phone. Our reason for providing this facility was based on personal experiences in the past where we have felt the need to post messages on our door from some remote location and so have had to phone through to a secretary or colleague and ask him or her to stick an appropriate post-it note message on our door. The typical message that a lecturer might ask to be posted would be something like "Sorry, car problems, away this A.M.", or "Sorry, running 30 mins late". Clearly such an approach disturbs the person being asked to write the post-it note and requires the person to be contactable (often not the case with very busy secretaries) and free to perform the task.

Sometimes, the message posted may also be posted to the computing department's mailing list, e.g. if the person has been taken ill and is likely to be off the entire day. However, for messages of slightly less importance (or less wide appeal) it is very unlikely that such an e-mail would be sent. During a typical office day, lecturers have a series of fixed appointments with students and also maintain a 'drop-in' period for students to discuss problems. A message such as "Running 30 mins late" is therefore 
extremely useful for supporting dynamic rescheduling and for reaching students that may not read their e-mail in time if an accompanying e-mail was actually sent.

\section{Experience and Analysis}

As Hermes has been deployed on a substantial scale and for a prolonged period we have been able to obtain significant feedback, both to enable evolutionary improvements and to give us a rich understanding of real use.

The remote messaging facility in Hermes (though not the overall Hermes system) was primarily envisioned as a feature to support lecturers in the department. Currently, six of the ten Hermes display owners are lecturers. Here are views of three of these lecturers (A, B and C) towards the remote messaging facility who have been long term Hermes users.

Lecturer $A$ attempted to use the remote messaging feature on a small number of occasions when the remote messaging feature was first introduced and unfortunately the reliability of the system was poor. He had found that the messages which he had sent using SMS had was not appeared on his Hermes display. This early experience damaged his trust of this aspect of the system and so he has subsequently not used this feature (although he very frequently posts messages on his Hermes display using the other approaches available). This lecturer is keen for the system to reassure him that a message has actually been displayed by providing him with improved feedback.

Lecturer $B$ has used the feature on several occasions. In common with lecturer A this lecturer started using the feature when it was first introduced and encountered some reliability problems. However, he has used the remote messaging feature successfully on several occasions and is prepared to trust the feature. In common with lecturer A, this lecturer is also keen for a greater level of feedback to be provided to increase his confidence that a sent message has successfully been displayed.

Lecturer $C$ was issued with a Hermes display several months later than lecturers A and $\mathrm{B}$ at a time when the reliability of the remote messaging feature was very high. Indeed, this lecturer has used the remote messaging feature fairly frequently for approximately six months and has not experienced any reliability problems with the SMS feature. Indeed, he has expressed a strong level of trust in the reliability of the remote messaging facility. Examples of his messages include "am running 20 mins late", "On bus 2.15 - in soon", "On bus - in shortly", "Gone to the gym", "Derek - in ww burger joint" and "In big q at post office.. Will be a bit late. C"

As can be seen, Lecturer C's messages include both temporal and location elements. Also, most messages are not targeted to a specific individual, with the exception of the message directed to Derek. We specifically asked the lecturer about his reasons for posting this targeted message and he revealed some interesting points.

It transpired that when the message was sent, lecturer $\mathrm{C}$ had an appointment with Derek in lecturer C's office but was delayed at a working lunch. He did not have Derek's mobile phone number, but, interestingly, he said he doubted whether he would have tried to contact Derek by mobile phone anyway (he suspected that Derek did not regularly carry a mobile phone). Also, by the time he realized he would be 
late, he doubted that Derek would have received an e-mail in time. Lecturer $\mathrm{C}$ also commented on the situated and "multipurpose" nature of his message, it provided not only the right notification to Derek but also informed those passing his office that he was currently delayed and likely to be running late. On this occasion situation, the SMS to his office door display was his primary means for communication.

\section{Discussion and Future Work}

In this paper, we have described the results of our initial exploration into a novel messaging paradigm, which focuses on enabling users to remotely update their (situated) office door displays. This has shown that:

- Trust and reliability are key issues for this use of messaging. Appropriate feedback is essential in order to establish confidence that the message has really been displayed and thus encourage continued use.

- This approach supplements existing email, voice and paper systems, but in some circumstances can become the primary means of communication.

- Traditionally, a lecturer might phone a secretary or colleague in order to ask him or her to place a post-it note message. As envisaged, Hermes has reduced this and so reduced disturbance, but in addition has encouraged messages such "In big $q$ at post office. . Will be a bit late" would probably not have warranted a special phone call to the secretary.

Our current development effort is focused on extending the current level of feedback provided by the Hermes system, answering one of the key problems highlighted above. The sender of a message will be provided with an acknowledgement when his or her SMS message is actually received by the door display as opposed to simply received by the SMS gateway (see figure 2).

One area of future work will be to explore the potential use of the Multimedia Messaging Service (MMS). This will allow the owner of a Hermes display to receive messages left on his or her door via an MMS capable mobile phone and also to enable the owner to send multimedia messages to display on the door display.

We also intend to trial the use of situated displays and remote messaging in the family home. One can envisage a situated display placed in the kitchen with other, more private, displays situated in other locations around the house, such as outside bedrooms. Family members would then be able to send messages to situated displays in addition to, the mobile phones of individual family members.

In summary, we believe that our initial studies suggest the significant potential of messaging to situated displays, as providing a simple, lightweight and flexible mechanism for displaying informal notifications to an appropriate location.

\section{References}

1. BBC News item: http://news.bbc.co.uk/1/hi/england/2784425.stm 
6 Keith Cheverst, Alan Dix, Dan Fitton, Adrian Friday and Marc Rouncefield

2. Cheverst, K., D. Fitton, and A. Dix. "Exploring The Evolution Of Office Door Displays", In K. O’Hara, M. Perry, E. Churchill, D. Russell (ed) Public and Situated Displays: Social and Interactional aspects of shared display technologies. To appear.

3. Mobile Data Association, http://www.mda-mobiledata.org/resource/hottopics/sms.asp

4. O'Hara, K. et al, Public, Community and Situated Displays: Design, use and interaction around shared information displays, Call for papers, available at: http://www.appliancestudio.com/cscw/cscwdisplayworkshopcall.htm (2002)

5. Weiser, M. and J. Seely Brown, "Designing Calm Technology," Chaper 6 "The Coming Age of Calm Technogy" in "Beyond Calculation - The Next Fifty Years of Computing" by P. J. Denning and R. M. Metcalfe, Copernicus/An Imprint of Springer-Verlag. 